Pacific Journal of Mathematic 


\title{
ON THE DIFFERENTIABILITY OF MULTIFUNCTIONS
}

\author{
F. S. DE Blasi
}

A new concept of differential for a multifunction is introduced. Here by a multifunction we mean a map from a Banach space $X$ to some specified family of non void subsets of a Banach space $Y$. The comparison with another definition due to Lasota and Strauss shows that if a multifunction admits both differentials, these must be equal. The results are applicable to the perturbation theory for multivalued differential equations in a Banach space

$$
\dot{x} \in F(x)
$$

in a neighborhood of a rest position.

1. Introduction. The concept of differentiability for multifunctions has been considered by many authors from different points of view ([1], [3], [8], [11], [13], [17], [18]). Of all these approaches, that developed by Lasota and Strauss [17] seems to be more useful in perturbation theory for ordinary differential equations in the real Euclidean space $R^{n}$. Further applications along this same direction were obtained in [10] (see also [9]). In the present paper, moving from an idea of Bridgland [3], a new concept of differentiability for a multifunction is studied. This notion seems to be useful in perturbation theory. In [7] an application to problems of stability for multivalued differential equations in Banach spaces is given.

The definitions and the main properties of a multivalued differential (i.e. the differential of a multifunction or, in particular, of a function) are contained in $\S \S 2$ and 3 . Now, it is perhaps better to start by giving an answer to the preliminary question: where one may encounter a multivalued differential. To this end we recall the well known Theorems 1.1 and 1.2, due to Lyapunov.

THEOREM 1.1 ([20] p. 222). Let $f: R^{n} \rightarrow R^{n}, f(0)=0$, be continuously differentiable in a neighborhood of the origin with Fréchet differential $f^{\prime}$ at the origin. Let all the eigenvalues of $f^{\prime}$ have negative real parts, i.e. the origin is asymptotically stable for

$$
\dot{x}=f^{\prime}(x) .
$$

Then the origin is asymptotically stable for

$$
\dot{x}=f(x) .
$$


Of the possible extensions of the above result we mention the following two:

(I) $f$ is single valued but not Fréchet differentiable at the origin. However $f$ has a multivalued differential $D$ at the origin, and so the variational equation which corresponds to (1.2) becomes

$$
\dot{x} \in D(x) \text {. }
$$

(II) $f$ is multivalued and admits at the origin a multivalued differential $D$. Thus, instead of (1.2) we have

$$
\dot{x} \in f(x),
$$

with corresponding variational equation (1.3).

In either case the problem arises whether the knowledge of a certain property of (1.3), for instance that the origin is a global attractor for this equation, implies that (1.2) (or (1.4)) possesses a similar, possibly weaker, property (see [17]).

THEOREM 1.2 ([20] p. 285). Let $f: R^{+} \times R^{n} \rightarrow R^{n}, R^{+}=[0, \infty)$, be continuously differentiable, periodic in $t$ with period $p>0$. Let the equation

$$
\dot{x}=f(t, x)
$$

have a periodic solution y of period $p$. Let all the characteristic numbers of the variational equation (along the periodic solution $y$ )

$$
\dot{x}=f^{\prime}(t, y(t)) x
$$

have moduli strictly less than 1 . Then the periodic solution y is asymptotically stable for (1.5).

A possible extension of Theorem 1.2 is the following:

(III) $f$ is single valued but not Fréchet differentiable along $y$. However $f$ has a multivalued differential $D$ at any point $(t, y(t))$. Thus (1.6) is replaced by

$$
\dot{x} \in D(t, y(t) ; x) .
$$

Then the problem arises whether, the fact that all solutions of (1.7) approach the origin for $t \rightarrow \infty$, implies that the periodic solution $y$ is asymptotically stable for (1.5) (see [10]).

In $\$ 2$ the definition of the multivalued differential $D_{x}$ for a multifunction is introduced. Several elementary consequences of this definition are reviewed in $\$ 3$. In the following one we consider, in infinite 
dimension, another definition of differential $\Delta_{x}$ for a multifunction. (This was introduced by Lasota and Strauss [17] for mappings from $R^{n}$ to $R^{n}$.) In Section 5 we consider $\gamma$-Lipschitz maps ( $\gamma$ is the Hausdorff measure of noncompactness [22]). Then we show that the multivalued differential of a $\gamma$-Lipschitz map, with constant $k$, is $\gamma$-Lipschitz with the same constant. In the subsequent paper [7] an application of the above theory to a problem of stability, by the first approximation method, for a multivalued differential equation in Banach space is presented.

2. Notation and preliminaries. Let $Y$ be a Banach space. For any $a \in Y$, define $S(a, r)=\{y:\|y-a\|<r\} \quad r \geq 0, \quad \bar{S}(a, r)=$ $\{y:\|y-a\| \leqq r\}, r \geqq 0$. We write $S, \bar{S}$ in place of $S(0,1), \bar{S}(0,1)$. Denote by: $\mathscr{B}(Y)\left(\right.$ resp. $\left.\mathscr{C}(Y), \mathscr{C}_{0}(Y), \mathscr{K}(Y), \mathscr{K}_{0}(Y)\right)$ the family of all non void bounded (resp. bounded closed, bounded closed convex, compact, compact convex) subsets of $Y ; N=\{1,2, \cdots\} ; \bar{A}$ the closure, $\overline{c o} A$ the closed convex hull of $A \subset Y$. Let $A, B \in B(Y)$. Define

$$
d(A, B)=\inf \{t>0: A \subset B+t S, B \subset A+t S\} .
$$

We review a number of well known properties of $d$, some of which will be used in the sequel. We have:

$$
\begin{aligned}
& d(A, B) \geqq 0, \quad d(A, A)=0 \\
& d(A, B)=d(B, A) \\
& d(A, B) \leqq d(A, C)+d(C, B) .
\end{aligned}
$$

To conclude that $d$ is a metric one has to show that $d(A, B)=0$ implies $A=B$. This is not true in $\mathscr{B}(Y)$, but it is in $\mathscr{C}(Y)$. The restriction of $d$ to couples of elements of $\mathscr{C}(Y)$ is called the Hausdorff metric in $\mathscr{C}(Y)$. We write $\|A\|$ in place of $d(A, 0)$.

The following lemma is fundamental.

Lemma 2.1 (Rådström [21]). Let $A, B, C$ be non void subsets of $Y$. Suppose $B$ closed and convex $C$ bounded and $A+C \subset B+C$. Then $A \subset B$.

Lemma 2.2. Let $A, A_{1}, B, B_{1} \in \mathscr{B}(Y)$. Then

(i) $\quad d(t A, t B)=t d(A, B) t \geqq 0$

(ii) $d\left(A+B, A_{1}+B_{1}\right) \leqq d\left(A, A_{1}\right)+d\left(B, B_{1}\right)$.

If $A, B \in \mathscr{C}_{0}(Y)$ and $C \in \mathscr{B}(Y)$ we have

(iii) $d(A+C, B+C)=d(A, B)$.

Proof. Property (i) is obvious. To prove (ii) let $t>d\left(A, A_{1}\right)$, $t_{1}>d\left(B, B_{1}\right)$. Then 


$$
\begin{array}{ll}
A \subset A_{1}+t S & B \subset B_{1}+t_{1} S \\
A_{1} \subset A+t S & B_{1} \subset B+t_{1} S
\end{array}
$$

and $A+B \subset A_{1}+B_{1}+\left(t+t_{1}\right) S, A_{1}+B_{1} \subset A+B+\left(t+t_{1}\right) S$ which imply $d\left(A+B, A_{1}+B_{1}\right) \leqq t+t_{1}$. Letting $t \rightarrow d\left(A, A_{1}\right), t_{1} \rightarrow d\left(B, B_{1}\right)$ we get (ii).

Let us prove (iii). By (ii) $d(A+C, B+C) \leqq d(A, B)$. Suppose the strict inequality holds and let $t$ be such that $d(A+C, B+C)<t<$ $d(A, B)$. Then

$$
\begin{aligned}
& A+C \subset B+C+t S \subset \overline{B+t S}+C \\
& B+C \subset A+C+t S \subset \overline{A+t S}+C
\end{aligned}
$$

and, since $\overline{B+t S}, \overline{A+t S}$ are closed convex while $C$ is bounded, Lemma 2.1 yields $A \subset \overline{B+t S}, B \subset \overline{A+t S}$. On the other hand

$$
\overline{B+t S}=\bigcap_{n=1}^{\infty}\left[(B+t S)+2^{-n} S\right], \quad \overline{A+t S}=\bigcap_{n=1}^{\infty}\left[(A+t S)+2^{-n} S\right],
$$

thus if we choose $n$ such that $t+2^{-n}<d(A, B)$ we obtain $A \subset B+\left(t+2^{-n}\right) S, B \subset A+\left(t+2^{-n}\right) S$. These imply $d(A, B) \leqq t+2^{-n}<$ $d(A, B)$, a contradiction. [8]).

Property (iii) is proved in [21] under different hypotheses (see also

Let $X, Y$ be Banach spaces. Let $U$ be a non void open subset of $X$.

Definition 2.3. $F: U \rightarrow \mathscr{B}(Y)$ is said to be upper semicontinuous (=u.s.c.) at $x \in U$ if for every $\epsilon>0$ there exists $\delta>0$ such that $F(x+h) \subset F(x)+\epsilon S$, when $\|h\|<\delta$. F is said to be continuous at $x$ if for every $\epsilon>0$ there exists $\delta>0$ such that $F(x+h) \subset F(x)+\epsilon S$ and $F(x) \subset$ $F(x+h)+\epsilon S$, when $\|h\|<\delta$.

Definition 2.4. $F: X \rightarrow \mathscr{B}(Y)$ is said to be homogeneous if $F(t x)=t F(x), t \geqq 0, x \in X$.

The following definition of differentiability is suggested by an idea due to Bridgland [3].

Definition 2.5. $F: U \rightarrow \mathscr{B}(Y)$ is said to be differentiable at $x \in U$ if there exist a map $D_{x}: X \rightarrow \mathscr{C}_{0}(Y)$, which is u.s.c. and homogeneous, and a number $\delta>0$ such that

$$
d\left(F(x+h), F(x)+D_{x}(h)\right)=o(h) \quad \text { when } \quad\|h\|<\delta .
$$


(Here $o(h)$ denotes a nonnegative function such that $\lim _{h \rightarrow 0} o(h) /\|h\|=$ 0 .) $D_{x}$ is called the (multivalued) differential of $F$ at $x$.

REMARK 2.6. Let $F$ be a map from $U$ to $\mathscr{K}_{0}(Y)$. In [18] Martelli and Vignoli define $F$ to be differentiable at $x \in U$ if there exists a map $S_{x}: X \rightarrow \mathscr{K}_{0}(Y)$, which is u.s.c. and homogeneous, and a number $\delta>0$ such that

$$
F(x+h)=F(x)+S_{x}(h)+R(h), \text { when }\|h\|<\delta,
$$

and $\lim _{h \rightarrow 0}\|R(h)\| /\|h\|=0$. We shall see later that the existence of $S_{x}$ implies that of $D_{x}$ and $S_{x}=D_{x}$. The converse is false. To see this define $F:(-\pi / 4, \pi / 4) \rightarrow \mathscr{K}_{0}(Y), Y=R^{n}$, by

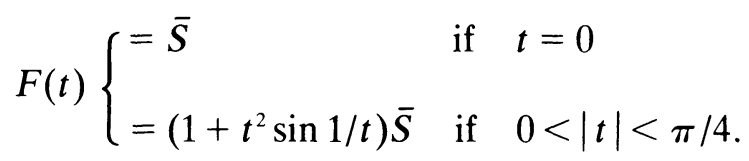

Then $F$ is differentiable at 0 and $D_{0}=0$. But $S_{0}$ does not exist for the existence of $S_{0}$ implies that, in a neighborhood of 0 the diameter of $F(t)$ is not less than the diameter of $F(0)$, which is clearly impossible.

3. Properties of differentiable multifunctions. In this section several elementary properties of differentiable multifunctions are reviewed. Let $U$ be a non void open subset of $X$. The following theorem shows that the differential $D_{x}$ is well defined.

THEOREM 3.1. The multivalued differential $D_{x}$ of $F: U \rightarrow \mathscr{B}(Y)$ at $x \in U$ if it exists is unique.

Proof. Let $\delta$ correspond to $D_{x}$. Let there exist $D_{x}^{1}$ and $\delta_{1}>0$ such that $d\left(F(x+h), F(x)+D_{x}^{1}(h)\right)=o^{1}(h)$, when $\|h\|<\delta_{1}$. Trivially $D_{x}(0)=$ $D_{x}^{1}(0)=0$, being both $D_{x}$ and $D_{x}^{1}$ homogeneous. Let $u \neq 0$. Let $t>0$ be such that $t\|u\|<\delta, \delta_{1}$. Then, by Lemma 2.2 (iii),

$$
\begin{aligned}
d\left(D_{x}(t u), D_{x}^{1}(t u)\right)= & d\left(D_{x}(t u)+F(x), D_{x}^{1}(t u)+F(x)\right) \\
\leqq & d\left(D_{x}(t u)+F(x), F(x+t u)\right) \\
& +d\left(F(x+t u), D_{x}^{1}(t u)+F(x)\right) \\
\leqq & o(t u)+o^{1}(t u) .
\end{aligned}
$$

Thus $\quad d\left(D_{x}(u), D_{x}^{1}(u)\right) \leqq o(t u) / t+o_{1}(t u) / t \quad$ and, letting $\quad t \rightarrow 0$, $d\left(D_{x}(u), D_{x}^{1}(u)\right)=0$. Since $D_{x}(u), D_{x}^{1}(u)$ are bounded closed we have $D_{x}(u)=D_{x}^{1}(u)$. 
REMARK 3.2. Suppose $F: U \rightarrow \mathscr{K}_{0}(Y)$ has the differential $S_{x}$. Then $D_{x}$ exists and $S_{x}=D_{x}$. In fact, if $\|h\|<\delta$,

$$
\begin{aligned}
d\left(F(x+h), F(x)+S_{x}(h)\right) \leqq & d\left(F(x+h), F(x)+S_{x}(h)+R(h)\right) \\
& +d\left(F(x)+S_{x}(h)+R(h), F(x)+S_{x}(h)\right) \\
\leqq & \|R(h)\|
\end{aligned}
$$

and, since $\lim _{h \rightarrow 0}\|R(h)\| /\|h\|=0$, we have $d\left(F(x+h), F(x)+S_{x}(h)\right)=$ $o(h)$. By the uniqueness of $D_{x}$ it follows $D_{x}=S_{x}$.

THEOREM 3.3. If $F: U \rightarrow \mathscr{B}(Y)$ is differentiable at $x$ it is there continuous.

Proof. Let $\epsilon>0$. Since $F$ is differentiable at $x$ there exists $\delta>0$ such that $d\left(F(x+h), F(x)+D_{x}(h)\right)=o(h)$, when $\|h\|<\delta$. Furthermore, since $D_{x}$ is u.s.c. at the origin and $D_{x}(0)=0$, there exists $0<\delta_{1}<\delta$ such that $D_{x}(h) \subset \epsilon S$ if $\|h\|<\delta_{1}$. For $\|h\|<\delta_{1}$ we have

$$
\begin{aligned}
d(F(x+h), F(x)) & \leqq d\left(F(x+h), F(x)+D_{x}(h)\right)+d\left(F(x)+D_{x}(h), F(x)\right) \\
& \leqq o(h)+\left\|D_{x}(h)\right\| \\
& \leqq o(h)+\epsilon
\end{aligned}
$$

and $F$ is continuous at $x$.

THEOREM 3.4. Let $U$ be a non void open and convex subset of $X$. The multifunction $F: U \rightarrow \mathscr{C}(Y)$ is constant if and only if, for every $x \in U$, $D_{x}=0$.

Proof. Let us prove the sufficiency of the condition (the necessity is trivial). For every $x \in U$ there exists $\delta>0$ such that $d(F(x+h), F(x))=$ $o(h)$ if $\|h\|<\delta$. Let $x, x_{1} \in U$. We have

$$
\begin{aligned}
& \left|d\left(F\left(x_{1}\right), F(x+h)\right)-d\left(F\left(x_{1}\right), F(x)\right)\right| \leqq d(F(x+h), F(x)) \\
& \left|d\left(F\left(x_{1}\right), F(x+h)\right)-d\left(F\left(x_{1}\right), F(x)\right)\right| /\|h\| \leqq o(h) /\|h\|, \quad 0<\|h\|<\delta .
\end{aligned}
$$

Let $h \rightarrow 0$. Then the real valued functional $x \mapsto d\left(F\left(x_{1}\right), F(x)\right)$, having zero Fréchet differential for every $x \in U$, must be constant. Since it vanishes for $x=x_{1}$ it is identically zero.

For $A, B \in \mathscr{C}(Y)$ define $d^{*}(A, B)=\inf \{t>0: A \subset B+t S\}$. We have: 


$$
\begin{aligned}
& d^{*}(A, B) \geqq 0, \quad d^{*}(A, B)=0 \quad \text { if and only if } A \subset B \\
& d^{*}(A, B) \leqq d^{*}(A, C)+d^{*}(C, B) \\
& d^{*}(A, B) \leqq d(A, B) .
\end{aligned}
$$

If $A=\{a\}, B=\{b\}$ then $d^{*}(A, B)=d(A, B)=\|a-b\|$.

Given a map $F: U \rightarrow \mathscr{C}(Y)$, a single valued function $f: U \rightarrow Y$ satisfying $f(x) \in F(x), x \in U$, is called a selection of $F$.

THEOREM 3.5. Let $F: X \rightarrow \mathscr{C}(Y), F(0)=0$, be differentiable at the origin with differential $D_{0}$. Let $f$ be a selection of $F$ in a neighborhood $S\left(0, \delta_{1}\right)$ of the origin of $X$. If $f$ has Fréchet differential $f_{0}^{\prime}$ at the origin then $f_{0}^{\prime}$ is a selection of $D_{0}$.

Proof. There exists $0<\delta<\delta_{1}$ such that

$$
d\left(F(h), D_{0}(h)\right)=o(h), \quad\left\|f(h)-f_{0}^{\prime}(h)\right\|=o^{1}(h) \quad \text { if } \quad\|h\|<\delta .
$$

Trivially $D_{0}$ and $f_{0}^{\prime}$ are equal for $u=0$. Let $u \neq 0$. Let $t>0$ be such that $t\|h\|<\delta$. Then we have

and

$$
\begin{aligned}
d^{*}\left(f_{0}^{\prime}(t u), D_{0}(t u)\right) \leqq & d^{*}\left(f_{0}^{\prime}(t u), f(t u)\right)+d^{*}(f(t u), F(t u)) \\
& +d^{*}\left(F(t u), D_{0}(t u)\right)
\end{aligned}
$$

$$
\begin{aligned}
d^{*}\left(f_{0}^{\prime}(u), D_{0}(u)\right) & \leqq t^{-1}\left\|f_{0}^{\prime}(t u)-f(t u)\right\|+t^{-1} d\left(F(t u), D_{0}(t u)\right) \\
& \leqq o^{1}(t u) / t+o(t u) / t .
\end{aligned}
$$

Letting $t \rightarrow 0$ we obtain $d^{*}\left(f_{0}^{\prime}(u), D_{0}(u)\right)=0$.

4. Comparison with another definition of differential. In [17] Lasota and Strauss gave the definition of a multivalued differential $\Delta_{x}$ for a single-valued map $F: R^{n} \rightarrow R^{n}$ and used such definition to prove a perturbation theorem for ordinary differential equations in $R^{n}$. Further results along this same direction were established in [10] and, for difference equations, in [9]. In this section the definition of $\Delta_{x}$ is extended to maps $F: X \rightarrow \mathscr{K}(Y)$, where $X, Y$ are Banach spaces. Furthermore the relationship between the multivalued differential $D_{x}$ and the Lasota-Strauss differential $\Delta_{x}$ is considered.

Let $X, Y$ be Banach spaces, $U \subset X$ be open and non void.

Definition 4.1. $\quad F: U \rightarrow \mathscr{K}(Y)$ is said to be Lipschitzian at $x \in U$ if $F(x)$ is singleton and there exist constants $L \geqq 0$ and $\delta>0$ such that $d(F(x+h), F(x)) \leqq L\|h\|$ if $\|h\|<\delta$. 
Definition 4.2. Let $F: U \rightarrow \mathscr{K}(Y)$ be Lipschitzian at $x \in U$. A map $\varphi: X \rightarrow \mathscr{K}_{0}(Y)$ is said to be an upper differential of $F$ at $x$ if $\varphi$ is u.s.c., homogeneous and there exists $\delta>0$ such that

$$
F(x+h) \subset F(x)+\varphi(h) \text { if }\|h\|<\delta .
$$

Denote by $\mathscr{F}$ the set of all upper differentials of $F$ at $x$. $\mathscr{F}$ may be empty. However, if $\operatorname{dim}(Y)<\infty, F$ has at least one upper differential, namely $\varphi(h)=L\|h\| \bar{S}$.

Definition 4.3. Let $F: U \rightarrow \mathscr{K}(Y)$ be Lipschitzian at $x \in U$. Suppose that $\mathscr{F} \neq \varnothing$ and, for each $h \in X, \bigcap_{\varphi \in \mathscr{F}} \varphi(h) \neq \varnothing$. Define the L. S. differential $\Delta_{x}: X \rightarrow \mathscr{K}_{0}(Y)$ by

$$
\Delta_{x}(h)=\bigcap_{\varphi \in \mathscr{F}} \varphi(h) \quad h \in X .
$$

The above definition reduces to that given by Lasota and Strauss [17] for single-valued maps $F: R^{n} \rightarrow R^{n}$.

REMARK 4.4. Berge [2] (p. 114) defines a map $F: U \rightarrow \mathscr{K}(Y)$ to be u.s.c at $x \in U$ if for every open set $G \supset F(x)$ there exists $\delta>0$ such that $F(x+h) \subset G$ if $\|h\|<\delta$. If $F$ is u.s.c. in this sense it is also u.s.c. according to Definition 2.3. Conversely let $F$ be u.s.c. at $x$. To prove that $F$ is u.s.c. according to Berge's definition it is sufficient to show the existence of a positive integer $n$ such that $F(x)+2^{-n} S \subset G$. Indeed, in the contrary case, for every $n \in N$, we have $\left(F(x)+2^{-n} S\right) \cap(Y \backslash G) \neq \varnothing$. This implies the existence of a sequence $\left\{y_{n}+s_{n}\right\}, y_{n} \in F(x), s_{n} \in 2^{-n} S$ such that $y_{n}+s_{n} \in Y \backslash G$. By the compactness of $F(x)$ we can and do assume, without loss of generality, $y_{n} \rightarrow y \in F(x)$. Since $y_{n}+s_{n} \rightarrow y$ and $Y \backslash G$ is closed, $y \in Y \backslash G$. From the contradiction the claim follows. Since ([2] p. 119) the intersection of any family of u.s.c. (homogeneous) mappings is u.s.c. (homogeneous) it remains proved that $\Delta_{x}$ if it exists is u.s.c. (homogeneous). Clearly for any $h \in X, \Omega(h)=\bigcap_{\varphi \in \mathscr{F}} \varphi(h)$ belongs to $\mathscr{K}_{0}(Y)$, provided it is non void. Thus the existence of $\Delta_{x}$ is finally established if we show that, for every $h \in X, \Omega(h) \neq \varnothing$.

THEOREM 4.5. Let $Y$ be reflexive. Let $F: U \rightarrow \mathscr{K}(Y)$ be Lipschitzian at $x \in U$. If $\mathscr{F} \neq \varnothing$ the L. S. differential $\Delta_{x}$ of $F$ at $x$ exists. Moreover $\Delta_{x}$ is u.s.c. and homogeneous.

Proof. After Remark 4.4 the only fact which requires a proof is that $\Omega(h) \neq \varnothing, h \in X$. Let $h \neq 0$ (the case $h=0$ is trivial). There exists a positive integer $k$ such that 


$$
\frac{d(F(x+h / n), F(x))}{\|h / n\|} \leqq L \quad \text { if } \quad n \geqq k
$$

Choose $y_{n} \in F(x+h / n)$. Since the sequence $\left\{\left(y_{n}-F(x)\right)\|h / n\|^{-1}\right\}_{n \geqq k}$ is bounded in the reflexive Banach space $Y$ we assume, without loss of generality that it converges weakly to some element $z \in Y$. Then

$$
z \in \overline{\operatorname{co}}\left\{\frac{y_{n}-F(x)}{\|h / n\|}\right\}_{n \geqq k} \subset \overline{\operatorname{co}}\left\{\frac{F(x+h / n)-F(x)}{\|h / n\|}\right\}_{n \geqq k} .
$$

Let $\varphi$ be any upper differential of $F$ at $x$ and let $\delta>0$ correspond. There exists $k_{1} \geqq k$ such that $n \geqq k_{1}$ implies $\|h / n\|<\delta$. For $n \geqq k_{1}$ we have $F(x+h / n) \subset F(x)+\varphi(h / n)$. Therefore

$$
z \in \overline{\operatorname{co}}\left\{\frac{\varphi(h / n)}{\|h / n\|}\right\}_{n \geqq k_{1}}=\varphi\left(\frac{h}{\|h\|}\right)
$$

and $\|h\| z \in \varphi(h)$. Since $\varphi$ is arbitrary $\|h\| z \in \Omega(h)$. omitted.

If $\operatorname{dim}(Y)<\infty$ the hypothesis $\mathscr{F} \neq \varnothing$ in the above theorem can be

Lemma 4.6. Let $X$ and $Y$ be separable Banach spaces. Let $F: U \rightarrow \mathscr{K}(Y)$ be Lipschitzian at $x$ with $L$. S. differential $\Delta_{x}$. Then there exists a sequence $\left\{\varphi_{n}\right\}$ of upper differentials of $F$ at $x$ such that

$$
\varphi_{n}(h) \supset \varphi_{n+1}(h), \quad \Delta_{x}(h)=\bigcap_{n=1}^{\infty} \varphi_{n}(h) \quad h \in X .
$$

Proof. Let $\Psi$ be any upper differential of $F$ at $x$. The graph $G_{\Psi}$ of $\Psi$ is closed for $\Psi$ is u.s.c. (Berge [2] p. 117). Since $X$ and $Y$ have countable bases, $X \times Y$ has the same property and, by Lindelöf theorem (Dunford and Schwartz [12] p. 12) there exists a sequence $\left\{\Psi_{n}\right\}$ of upper differentials such that $\bigcap_{\varphi \in \mathscr{F}} G_{\varphi}=\bigcap_{n=1}^{\infty} G_{\Psi_{n}}$. Then

$$
G_{\Delta_{x}}=\bigcap_{\varphi \in \mathscr{F}} G_{\varphi}=\bigcap_{n=1}^{\infty} G_{\Psi_{n}}=G_{\cap_{n=1}^{x} \Psi_{n}}
$$

implies $\Delta_{x}=\bigcap_{n=1}^{\infty} \Psi_{n}$. Since a finite intersection of u.s.c. (homogeneous) maps is u.s.c. (homogeneous) the sequence $\left\{\varphi_{n}\right\}, \varphi_{n}=\bigcap_{k=1}^{n} \Psi_{k}$ consists of upper differentials which satisfy the conclusions of the lemma.

The following result is useful in perturbation theory [10]. 
THEOREM 4.7. Let $X, Y$ be finite dimensional Banach spaces. Let $F: U \rightarrow \mathscr{K}(Y)$ be Lipschitzian at $x \in U$, with constant L. Let $\Delta_{x}: X \rightarrow \mathscr{K}_{0}(Y)$ be continuous. Then the map $V_{\epsilon}: h \mapsto \Delta_{x}(h)+\epsilon\|h\| \bar{S}$, $\epsilon>0$, is an upper differential of $F$ at $x$.

Proof. The map $V_{\epsilon}$ from $X$ to $\mathscr{K}_{0}(Y)$ is continuous and homogeneous. To conclude that $V_{\epsilon}$ is an upper differential of $F$ at $x$ we need to show that there exists $\delta>0$ such that $F(x+h) \subset F(x)+V_{\epsilon}(h)$ if $\|h\|<\delta$. Suppose the contrary. There exists a sequence $\left\{h_{n}\right\}, h_{n} \neq 0, h_{n} \rightarrow 0$ such that $F\left(x+h_{n}\right) \not \subset F(x)+V_{\epsilon}\left(h_{n}\right)$. Thus there exists a sequence $\left\{y_{n}\right\}, y_{n} \in$ $F\left(x+h_{n}\right)$, satisfying $y_{n}-F(x) \notin V_{\epsilon}\left(h_{n}\right)$ or, equivalently,

$$
\left(y_{n}-F(x)\right) /\left\|h_{n}\right\| \notin V_{\epsilon}\left(h_{n} /\left\|h_{n}\right\|\right) \quad n \in N .
$$

Since $\left\{h_{n} /\left\|h_{n}\right\|\right\}$ and $\left\{\left(y_{n}-F(x)\right) /\left\|h_{n}\right\|\right\}$ are bounded and $X, Y$ are finite dimensional, we can and do assume (without loss of generality)

$$
h_{n} /\left\|h_{n}\right\| \rightarrow h \in X, \quad\left(y_{n}-F(x)\right) /\left\|h_{n}\right\| \rightarrow y \in Y .
$$

Suppose $y \in \Delta_{x}(h)+(\epsilon / 2) \bar{S}$. This implies $y+(\epsilon / 4) \bar{S} \subset \Delta_{x}(h)+$ $(3 / 4) \epsilon \bar{S}$ and for $n$ sufficiently large, say $n \geqq k$, $\left(y_{n}-F(x)\right) /\left\|h_{n}\right\| \in \Delta_{x}(h)+(3 / 4) \epsilon \bar{S}$. Since $\Delta_{x}$ is continuous at $h$ there exists $k_{1} \geqq k$ such that $\Delta_{x}(h) \subset \Delta_{x}\left(h_{n} /\left\|h_{n}\right\|\right)+(\epsilon / 4) \bar{S}$ if $n \geqq k_{1}$. Thus

$$
\left(y_{n}-F(x)\right) /\left\|h_{n}\right\| \in \Delta_{x}\left(h_{n} /\left\|h_{n}\right\|\right)+\epsilon \bar{S}=V_{\epsilon}\left(h_{n} /\left\|h_{n}\right\|\right)
$$

if $n \geqq k_{1}$, a contradiction.

Suppose $y \notin \Delta_{x}(h)+(\epsilon / 2) \bar{S}$. Then if $\epsilon_{1}$ is such that $0<\epsilon_{1}<\epsilon / 2$ we have $\bar{S}\left(y, \epsilon_{1}\right) \cap \Delta_{x}(h)=\varnothing$. By Lemma 4.6 there exists a sequence $\left\{\varphi_{m}\right\}$ of upper differentials of $F$ at $x$ satisfying (4.1).

We claim that there is $k \in N$ such that for all $m \geqq k$ we have $\bar{S}\left(y, \epsilon_{1}\right) \cap \varphi_{m}(h)=\varnothing$. Let the claim be false. Since $\varphi_{1}(h) \supset \varphi_{2}(h) \supset \cdots$, for every $m \in N$ there exists $z_{m}$ in both sets $\bar{S}\left(y, \epsilon_{1}\right)$ and $\varphi_{m}(h)$. Without loss of generality we assume $z_{m} \rightarrow z$. Then $z \in \bar{S}\left(y, \epsilon_{1}\right)$ and $z \in \varphi_{m}(h)$ for every $m \in N$, thus $z \in \bar{S}\left(y, \epsilon_{1}\right) \cap \Delta_{x}(h)$, a contradiction. The claim is true. This implies

$$
\bar{S}\left(y, \frac{\epsilon_{1}}{2}\right) \cap\left(\varphi_{m}(h)+\frac{\epsilon_{1}}{2} \bar{S}\right)=\varnothing, \quad m \geqq k
$$

By (4.2), for all $n$ sufficiently large say $n \geqq r$ we have

$$
\left(y_{n}-F(x)\right) /\left\|h_{n}\right\| \in \bar{S}\left(y, \frac{\epsilon_{1}}{2}\right), \quad \varphi_{m}\left(h_{n} /\left\|h_{n}\right\|\right) \subset \varphi_{m}(h)+\frac{\epsilon_{1}}{2} \bar{S}
$$


and so, by virtue of (4.3), $\left(y_{n}-F(x)\right) /\left\|h_{n}\right\| \notin \varphi_{m}\left(h_{n} /\left\|h_{n}\right\|\right)$ i.e. $y_{n} \notin F(x)+$ $\varphi_{m}\left(h_{n}\right)$ for all $n \geqq r$. This implies $F\left(x+h_{n}\right) \not \subset F(x)+\varphi_{m}\left(h_{n}\right), n \geqq r$, a contradiction since $\varphi_{m}$ is an upper differential of $F$.

Next theorem shows that $D_{x}=\Delta_{x}$ if both exist.

THEOREM 4.8. Let $X, Y$ be Banach spaces. Let $F: U \rightarrow \mathscr{K}_{0}(Y)$ be Lipschitzian at $x \in U \subset X$. Then $\Delta_{x}=D_{x}$ if both exist.

Proof. By hypothesis there exists $\delta>0$ such that $d\left(F(x+h), F(x)+D_{x}(h)\right)=o(h)$ if $\|h\|<\delta$. Let $\epsilon>0$. Then there exists $0<\delta_{1}<\delta$ such that

$$
\begin{aligned}
F(x+h) & \subset F(x)+D_{x}(h)+\epsilon\|h\| \bar{S} \\
F(x)+D_{x}(h) & \subset F(x+h)+\epsilon\|h\| \bar{S} \text { if }\|h\|<\delta_{1} .
\end{aligned}
$$

Let $\varphi$ be any upper differential of $F$. This implies the existence of $0<\delta_{2}<\delta_{1}$ such that $F(x+h) \subset F(x)+\varphi(h)$, if $\|h\|<\delta_{2}$. Then $F(x+h)+\epsilon\|h\| \bar{S} \subset F(x)+\varphi(h)+\epsilon\|h\| \bar{S}$ and, by (4.5), $F(x)+D_{x}(h) \subset$ $F(x)+\varphi(h)+\epsilon\|h\| \bar{S}$, if $\|h\|<\delta_{2}$. Thus, $D_{x}(h) \subset \varphi(h)+\epsilon\|h\| \bar{S}$ from which one easily obtains $D_{x}(h) \subset \varphi(h)$, if $\|h\|<\delta_{2}$. Since $\varphi$ is any upper differential of $F$ we have $D_{x}(h) \subset \Delta_{x}(h)$ and, by the homogeneity of $D_{x}$ and $\Delta_{x}$, the inclusion holds for all $h \in X$.

Next let us show the reverse inclusion. Let $\varphi$ be any upper differential of $F$. Define $\varphi_{1}(h)=\varphi(h) \cap\left(D_{x}(h)+\epsilon\|h\| \bar{S}\right), h \in X$. We claim that $\varphi_{1}$ is an upper differential of $F$. From (4.4) and $F(x+h) \subset$ $F(x)+\varphi(h)$, which hold for $\|h\|$ small enough, it follows that $\varphi_{1}(h) \neq \varnothing$ in a neighborhood of the origin and, by homogeneity, for all $h \in X$. Trivially $\varphi_{1}(h)$ is convex, for every $h \in X$. Furthermore, for each $h \in X$, $D_{x}(h)$ is compact, for it is contained in $\Delta_{x}(h)$, and so $\varphi_{1}(h)$ is compact being the intersection of $\varphi(h)$ compact, and $D_{x}(h)+\epsilon\|h\| \bar{S}$ closed. Thus $\varphi_{1}$ maps $X$ into $\mathscr{K}_{0}(Y)$. Clearly $\varphi_{1}$ is homogeneous and satisfies $F(x+h) \subset F(x)+\varphi_{1}(h)$, for $\|h\|$ sufficiently small. So to conclude that $\varphi_{1}$ is an upper differential of $F$ it remains to be shown that it is u.s.c. But this follows at once from a result of Berge ([2] p. 117) because the map $h \mapsto D_{x}(h)+\epsilon\|h\| \bar{S}$ from $X$ to $\mathscr{C}_{0}(Y)$ is closed and $\varphi: X \rightarrow \mathscr{K}_{0}(Y)$ is u.s.c. Then there exists $\delta_{3}>0$ such that $\Delta_{x}(h) \subset \varphi_{1}(h) \subset D_{x}(h)+$ $\epsilon\|h\| \bar{S}$, if $\|h\|<\delta_{3}$, which implies $\Delta_{x}(h /\|h\|) \subset D_{x}(h /\|h\|), 0<\|h\|<\delta_{3}$. By homogeneity, $\Delta_{x}(h) \subset D_{x}(h)$ for every $h \in X$.

5. The differential of a $\gamma$-Lipschitz function. In this section it is shown that the differential $D_{x}$ of a multifunction which is $\gamma$-Lipschitz with constant $k$ possesses this same property. Let us introduce the following 
Definition 5.1. Let $A \in \mathscr{B}(Y)$. The measure $\gamma(A)$ of noncompactness of $A$ is defined by

$$
\gamma(A)=\inf \{t>0: \text { there exists } C \in \mathscr{K}(Y) \text { such that } A \subset C+t \bar{S}\} .
$$

There are alternative (non equivalent) definitions of measures of noncompactness ([5], [14], [16], [22]). That which we use seems to be flexible enough to be adapted for the measure of noncompactness in the weak topology as well [6]. The following theorem is well known. However we include the proofs of those statements which are proved in a different, perhaps simpler, way (see (f)-(i)).

THEOREM 5.2. The functional $\gamma$ has the properties:

(a) $A \subset B$ implies $\gamma(A) \leqq \gamma(B)$

(b) $\gamma(A)=\gamma(\bar{A})$

(c) $\gamma(A)=0$ if and only if $\bar{A}$ is compact

(d) $\gamma(A+B) \leqq \gamma(A)+\gamma(B)$

(e) $\gamma(s A)=s \gamma(A) \quad s \geqq 0$

(f) $\gamma(A)=\gamma(\overline{\mathrm{co}} A)$

(g) $\quad \gamma\left(\cup_{u \in[0, s]} u A\right)=s \gamma(A)$

(h) $\gamma(S)=1$ if $\operatorname{dim}(Y)=\infty$

(i) $\gamma(A+B)=\gamma(A)$ if $\gamma(B)=0$.

Proof. (a)-(e) follow easily from the Definition 5.1. (f) Let $\epsilon>0$. There exist $\gamma(A)<t<\gamma(A)+\epsilon$ and $C \in \mathscr{K}(Y)$ such that $A \subset C+t \bar{S}$. This implies $A \subset \overline{c o} C+t \bar{S}$ where, by Mazur theorem (Dunford and Schwartz [12] p. 416), $\overline{\mathrm{co}} C$ is compact. Thus $\overline{\mathrm{co}} A \subset \overline{\mathrm{co}} C+t \bar{S}$, being the second member convex and closed. The last inclusion shows $\gamma(\overline{\mathrm{co}} A) \leqq t$ and $\gamma(\overline{\operatorname{co}} A) \leqq \gamma(A)$. The reverse inequality is trivial.

(g) Let $\epsilon>0$. There exist $\gamma(A)<t<\gamma(A)+\epsilon$ and $C \in \mathscr{K}(Y)$ such that $A \subset C+t \bar{S}$. This implies $A \subset(C \cup\{0\})+t \bar{S} \subset C_{1}+t \bar{S}$ where $C_{1}=\overline{\mathrm{co}}(C \cup\{0\})$ is compact. Thus, for every $u \in[0, s], u A \subset u C_{1}+u t \bar{S} \subset$ $s C_{1}+s t \bar{S}$ (since $C_{1}$ is convex and contains the origin) and we have $\bigcup_{u \in[0, s]} u A \subset s C_{1}+s t \bar{S}$. This implies $\quad \gamma\left(\bigcup_{u \in[0, s]} u A\right) \leqq s t \quad$ and $\gamma\left(\cup_{u \in[0, s]} u A\right) \leqq s \gamma(A)$. The reverse inequality is obvious.

(h) Since $\bar{S}=\{0\}+1 \bar{S}$ we have $\gamma(\bar{S}) \leqq 1$. Suppose $\gamma(\bar{S})<1$. Then there exist $\gamma(\bar{S})<t<1$ and $C \in \mathscr{K}(Y)$ such that $\bar{S} \subset C+t \bar{S}$. From this

$$
\begin{aligned}
& \bar{S} \subset \overline{\mathrm{CO}} C+t \bar{S} \\
& (1-t) \bar{S}+t \bar{S} \subset \overline{\mathrm{CO}} C+t \bar{S}
\end{aligned}
$$

and, by Lemma 2.1, $(1-t) \bar{S} \subset \overline{c o} C$. Thus $\bar{S} \subset(1-t)^{-1} \overline{c o} C$ and since the 
set on the right is compact such must be $\bar{S}$. This is a contradiction since $\operatorname{dim}(Y)=\infty$.

(i) Let $b \in B$. Then $A \subset A+B+\{-b\}$ implies

$$
\gamma(A) \leqq \gamma(A+B+\{-b\})=\gamma(A+B) \leqq \gamma(A)+\gamma(B)=\gamma(A)
$$

and (i) is true.

Denote by $U$ a non void open subset of $Y$.

Definition 5.3. $F: U \rightarrow \mathscr{K}(Y)$ is said to be $\gamma$-Lipschitz, with constant $k \geqq 0$, if for every $A \in \mathscr{B}(Y), A \subset U$, we have $\gamma(F(A)) \leqq$ $k \gamma(A)$.

Now we want to show that the multivalued differential of a $\gamma$ Lipschitz map is $\gamma$-Lipschitz, with the same constant.

THEOREM 5.4. Let $F: U \rightarrow \mathscr{K}(Y)$ be $\gamma$-Lipschitz with constant $k$. Let $D_{x}$ be the differential of $F$ at $x \in U$. Then $D_{x}$ is $\gamma$-Lipschitz with the same constant $k$.

Proof. There exists $\delta>0$ such that $d\left(F(x+h), F(x)+D_{x}(h)\right)=$ $o(h)$ if $\|h\|<\delta$. This implies

$$
F(x)+D_{x}(h) \subset F(x+h)+\left(o(h)+\|h\|^{2}\right) S \quad \text { if } \quad\|h\|<\delta .
$$

Let $A \in \mathscr{B}(Y), A \subset U$. Let $t>0$ be such that $t\|A\|<\delta$. Let $\sigma(t)=$ $\sup \{o(h): h \in t A\}$. It is easy to see that $\lim _{t \rightarrow 0} \sigma(t) / t=0$. Let $h \in t A$. We have

$$
\begin{aligned}
& F(x)+D_{x}(h) \subset F(x+t A)+\left[\sigma(t)+t^{2}\|A\|^{2}\right] S \\
& F(x)+D_{x}(t A) \subset F(x+t A)+\left[\sigma(t)+t^{2}\|A\|^{2}\right] S .
\end{aligned}
$$

Using the properties of $\gamma$

$$
\begin{aligned}
\gamma\left(D_{x}(t A)\right) & =\gamma\left(F(x)+D_{x}(t A)\right) \\
& \leqq \gamma(F(x+t A))+\sigma(t)+t^{2}\|A\|^{2} \\
& \leqq k \gamma(t A)+\sigma(t)+t^{2}\|A\|^{2} .
\end{aligned}
$$

Thus

$$
\gamma\left(D_{x}(A)\right) \leqq k \gamma(A)+\sigma(t) / t+t\|A\|^{2}
$$

and, letting $t \rightarrow 0$, the desired result follows. 
Corollary 5.5 (Daneš [4], Nussbaum [19], Sadovskiǐ [22]). Let $F: U \rightarrow Y$ be a single valued $\gamma$-Lipschitz map with constant $k$. Let $F_{x}^{\prime}$ be the Fréchet differential of $F$ at $x \in U$. Then $F_{x}^{\prime}$ is $\gamma$-Lipschitz with the same constant $k$.

Definition 5.6. Let $U=\{x \in X:\|x\|>r\}, r>0 . F: U \rightarrow \mathscr{B}(Y)$ is said to be differentiable at infinity if there exist a map $D_{\infty}: X \rightarrow \mathscr{C}_{0}(Y)$, which is u.s.c. and homogeneous, and a number $\delta>r$ such that

$$
d\left(F(x), D_{x}(x)\right)=o(x) \text { when }\|x\|>\delta
$$

and $\lim _{x \rightarrow \infty} o(x) /\|x\|=0 . D_{x}$ is called the asymptotic differential of $F$.

Definition 5.7. (Krasnosel'skiǐ [15] p. 207). Let $F: U \rightarrow Y$ be a continuous single valued map, $U$ being as in the above definition. Let there exist a linear map $F_{x}^{\prime}$ and a number $\delta>r$ such that $F(x)=$ $F_{x}^{\prime}(x)+z(x)$, if $\|x\|>\delta$, and $\lim _{x \rightarrow \infty} z(x) /\|x\|=0$. Then $F$ is said to be asymptotically linear and $F_{\infty}^{\prime}$ is called the asymptotic derivative of $F$.

THEOREM 5.8. The asymptotic differential $D_{\infty}$ of $F: U \rightarrow \mathscr{B}(Y)$ if exists is unique.

Proof. Similar to that of Theorem 3.1.

THEOREM 5.9. Let $U=\{y \in Y:\|y\|>r\}, r>0$. Let $F: U \rightarrow \mathscr{K}(Y)$ be $\gamma$-Lipschitz with constant $k$. Let $D_{\infty}$ be the asymptotic differential of $F$. Then $D_{x}$ is $\gamma$-Lipschitz with the same constant $k$.

Proof. Similar to that of Theorem 5.4.

Since a single valued continuous map is completely continuous if and only if it is $\gamma$-Lipschitz with constant $k=0$, we have

COROLlaRY 5.10 (Krasnosel'skii [15] p. 207). The asymptotic derivative $F_{x}^{\prime}$ of a completely continuous single valued map $F: U \rightarrow Y$ is completely continuous.

\section{REFERENCES}

1. H. T. Banks and M. Q. Jacobs, A differential calculus for multifunctions, J. Math. Anal. Appl., 29 (1970), 246-272.

2. C. Berge, Espaces topologiques et fonctions multivoques, Dunod, Paris, 1966.

3. T. F. Bridgland, Trajectory integrals of set valued functions, Pacific J. Math., 33 (1970), 43-67.

4. J. Daneš, Some fixed point theorems in metric and Banach spaces, Comment. Math. Univ. Carolinae, 12 (1971), 37-51. 
5. — On the Istrătescu's measure of noncompactness, Bull. Math. Soc. Sci. Math. R. S. Roumanie, 16 (64) (1972), 403-406.

6. F. S. De Blasi, On a property of the unit sphere in a Banach space (submitted).

7. - Existence and stability of solutions for autonomous multivalued differential equations in Banach space, Atti Acc. Naz. Lincei, Rend. Cl. Sc. Math. Nat. (to appear).

8. F. S. De Blasi and F. Iervolino, Equazioni differenziali con soluzioni a valore compatto convesso, Boll. Un. Mat. Ital., 4 (1969), 491-501.

9. F. S. de Blasi and J. Schinas, Exponential stability of difference equations which cannot be linearized, Atti Acc. Naz. Lincei, Rend. Cl. Sc. Mat. Nat., 54 (1973), 16-21.

10. - The Lyapunov theorem on the behavior of solutions of a non autonomous differential system in a neighborhood of a periodic solution with corresponding multivalued variational equation, $\mathrm{J}$. Differential Equations, (to appear).

11. G. Debreu, Integration of correspondences, in Proc. Fifth Berkeley Symposium on Mathematical Statistics and Probability, Vol. II, part 1, p. 351-372, University of California Press, Los Angeles, 1967.

12. N. Dunford and J. T. Schwartz, Linear Operators, part I, Wiley (Interscience), New York, 1959.

13. M. Hukuhara, Intégration des applications mesurables dont la valeur est un compact convexe, Funkcial. Ekvac., 10 (1967), 205-22.3.

14. V. I. Istrăţescu, On a measure of noncompactness, Bull. Math. Soc. Sci. Math. R. S. Roumanie, 16 (64) (1972), 195-197.

15. M. Krasnosel'skiǐ, Topological Methods in the Theory of Nonlinear Integral Equations, Pergamon Press, London, 1963.

16. C. Kuratowski, Sur les espaces complets, Fund. Math., 15 (1930), 301-309.

17. A. Lasota and A. Strauss, Asymptotic behavior for differential equations which cannot be locally linearized, J. Differential Equations, 10 (1971), 152-172.

18. M. Martelli and A. Vignoli, On differentiability of multi-valued maps, Boll. Un. Mat. Ital., (4) 10 (1974), 701-712.

19. R. D. Nussbaum, The fixed point index and fixed point theorems for $k$-set contractions, Ph. D. Thesis, University of Chicago, 1969.

20. L. Pontryagin, Équations différentielles ordinaires, Éditions MIR, Moscow, 1969.

21. H. Rådström, An embedding theorem for spaces of convex sets, Proc. Amer. Math. Soc., 3 (1952), $165-169$.

22. B. N. Sadovskiǐ, Limit-compact and condensing operators, Uspehi Math Nauk, 271 (1972), (in Russian). Eng. Trans., Russian Math. Survey, p. 85-155.

Received July 17, 1975 and in revised form February 12, 1976.

Istituto Matematico "U. DinI"

Viale Morgagni 67/A

ITALIA 




\section{Pacific Journal of Mathematics}

\section{Vol. 66, No. $1 \quad$ November, 1976}

Helen Elizabeth. Adams, Factorization-prime ideals in integral domains ............ Patrick Robert Ahern and Robert Bruce Schneider, The boundary behavior of Henkin's kernel.

Daniel D. Anderson, Jacob R. Matijevic and Warren Douglas Nichols, The Krull

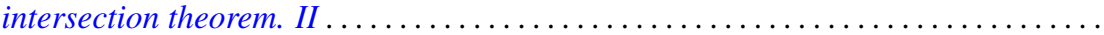

Efraim Pacillas Armendariz, On semiprime P.I.-algebras over commutative regular

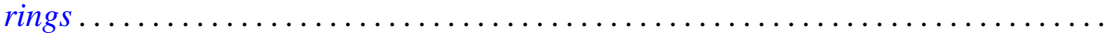

Robert H. Bird and Charles John Parry, Integral bases for bicyclic biquadratic fields

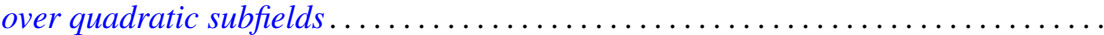

Tae Ho Choe and Young Hee Hong, Extensions of completely regular ordered

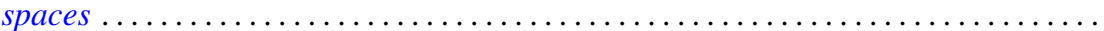

John Dauns, Generalized monoform and quasi injective modules ...............

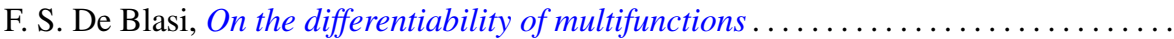

Paul M. Eakin, Jr. and Avinash Madhav Sathaye, R-endomorphisms of $R[[X]]$ are

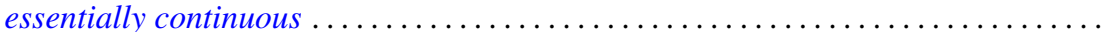

Larry Quin Eifler, Open mapping theorems for probability measures on metric

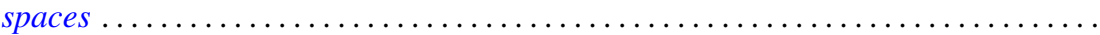

Garret J. Etgen and James Pawlowski, Oscillation criteria for second order self adjoint

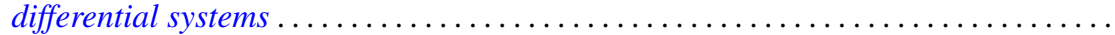

Ronald Fintushel, Local $S^{1}$ actions on 3-manifolds .

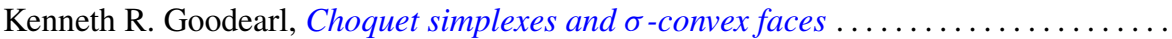

John R. Graef, Some nonoscillation criteria for higher order nonlinear differential

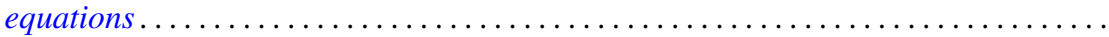

Charles Henry Heiberg, Norms of powers of absolutely convergent Fourier series: an example.

Les Andrew Karlovitz, Existence of fixed points of nonexpansive mappings in a space

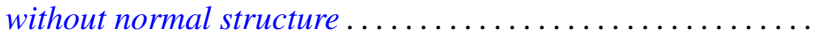

Gangaram S. Ladde, Systems of functional differential inequalities and functional

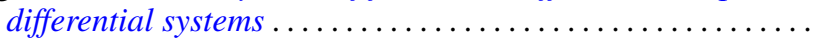

Joseph Michael Lambert, Conditions for simultaneous approximation and interpolation with norm preservation in $C[a, b]$.

Ernest Paul Lane, Insertion of a continuous function.

Robert F. Lax, Weierstrass points of products of Riemann surfaces .

Dan McCord, An estimate of the Nielsen number and an example concerning the Lefschetz fixed point theorem...

Paul Milnes and John Sydney Pym, Counterexample in the theory of continuous functions on topological groups...

Peter Johanna I. M. De Paepe, Homomorphism spaces of algebras of holomorphic functions

Judith Ann Palagallo, A representation of additive functionals on $L^{p}$-spaces,

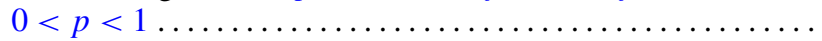

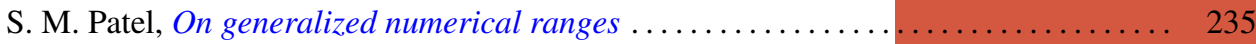

Thomas Thornton Read, A limit-point criterion for expressions with oscillatory

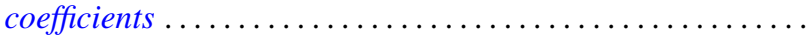

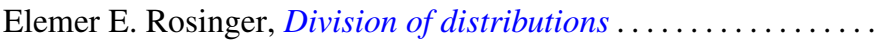

Peter S. Shoenfeld, Highly proximal and generalized almost finite

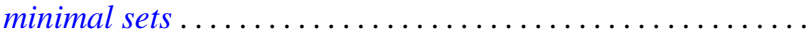

R. Sirois-Dumais and Stephen Willard, Quotient-universal sequential spaces

Robert Charles Thompson, Convex and concave functions of singular values of matrix sums....

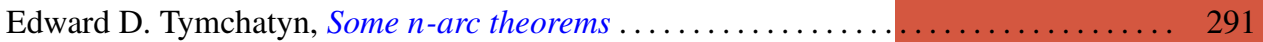

